\title{
Melanin and Hemoglobin Identification for Skin Disease Analysis
}

\author{
Zhao Liu, Josiane Zerubia \\ Ayin Research Group, INRIA Sophia Antipolis Méditerranée, France (https://team.inria.fr/ayin/) \\ Zhao.Liu@inria.fr, Josiane.Zerubia@inria.fr
}

\begin{abstract}
This paper proposes a novel method to extract melanin and hemoglobin concentrations of human skin, using bilateral decomposition with the knowledge of a multiple layered skin model and absorbance characteristics of major chromophores. Different from state-of-art approaches, the proposed method enables to address highlight and strong shading usually existing in skin color images captured under uncontrolled environment. The derived melanin and hemoglobin indices, directly related to the pathological tissue conditions, tend to be less influenced by external imaging factors and are effective for describing pigmentation distributions. Experiments demonstrate the value of the proposed method for computer-aided diagnosis of different skin diseases. The diagnostic accuracy of melanoma increases by $9-15 \%$ for conventional RGB lesion images, compared to techniques using other color descriptors. The discrimination of inflammatory acne and hyperpigmentation reveals acne stage, which would be useful for acne severity evaluation. It is expected that this new method will prove useful for other skin disease analysis.
\end{abstract}

Keywords-melanin identification; hemoglobin identification; bilateral decomposition; skin image analysis

\section{INTRODUCTION}

Skin color is an important characteristic for accurate diagnosis and grading of cutaneous lesions by experienced dermatologists in clinical practice. For example, the presence of multiple color shades and pigmentation asymmetry within lesions often indicates a high risk of developing melanoma. However, visual perception of skin color is not only credited to major chromophores (melanin and hemoglobin) underneath the skin surface, but is also affected by external illumination and spectral responses of imaging detectors. Skin color representation in a specific color space (e.g. RGB and its transformations) is not a genuine physical quantity. It derives from color matching functions of human visual system [1]. It sometimes fails to provide precise information about the concentrations of cutaneous chromophores, and is easily influenced by external imaging factors. Accordingly the direct use of conventional colorimetry may not properly describe the histological content of skin and tend to yield less trustworthy results when applied for skin disease analysis.

A number of studies have been developed for noninvasive assessment of melanin and hemoglobin. Tsumura et al. [2] applied independent component analysis (ICA) on RGB skin image to separate average concentrations of melanin and hemoglobin. Madooei et al. [3] extended their work by introducing extra factors to spectral reflection of skin model to reduce external effects such as shading and sensor characteristics. These methods show effectiveness of describing skin coloration for melanoma diagnosis. However, ICA-based methods are only responsible for separating skin image into two independent components, and do not associate the knowledge of absorbance spectrum. This results in ambiguities when differentiating melanin from hemoglobin.

Claridge et al. [4] proposed a sophisticated multilayered skin model using Kubelka-Munk theory for the extraction of epidermal and dermal melanin, blood, and collagen thickness using multispectral skin data. Yamamoto et al. [5] applied a much simple three layered skin model based on Lambert's law, and calculated melanin index and erythema index from RGB skin images. These methods well discriminate melanin and hemoglobin concentrations. But Claridge's approach requires multispectral images which are not always available in clinical practice, while Yamamoto's algorithm is reported sensitive to imaging circumstantial conditions [5].

More importantly, all of the above methods either do not have/specify an illumination and shading removal step $[2,4,5]$, or cancel out the effects of illumination and shading by directly dividing two spectral responses, provided that there is the same amount of external artifacts embedded in different spectral images [3]. But illumination spectrum varies with wavelength. Direct division fails to address large dynamic range intensity (e.g. highlight, shading) in skin images captured under uncontrolled conditions.

This paper proposes a novel chromophore extraction method using bilateral decomposition, which removes the effects of external imaging factors through a nonlinear weighted average process. Incorporating the knowledge of chromophore absorption, melanin and hemoglobin densities are well identified. Experiments show the value of this new method for the application to different skin disease analysis.

\section{OPTICS OF SKIN}

Human skin can be simplified as a thin structure with distinctive multiple layers, which correspond to melanincontaining epidermis, superficial vascular plexus dermis, and subcutis with collagen and fat [5]. Based on this multilayered model, previous methods [2, 3] use Lambert-Beer law for skin radiance provided that there is little effect of specular reflection. This diffuse reflectance model is effective in dealing with dermoscopy images under polarized lighting, but it is hard to handle conventional RGB skin images captured in uncontrolled imaging setting. The present study thus considers specular reflection and diffuse reflection simultaneously, and skin image intensity $I$ at pixel $x$ and wavelength $\lambda$ can be represented by a combination of specular reflection $I_{s}$ and diffuse reflection $I_{d}$ as: 


$$
\begin{aligned}
& I(x, \lambda)=I_{S}(x, \lambda)+I_{d}(x, \lambda) \\
& =Q(\lambda)\left[E_{S}(\lambda)\left(w_{s}(x)\right)^{\alpha}+E_{d}(\lambda) w_{d}(x) e^{-2\left(\mu_{m}^{\lambda} l_{m}^{\lambda} c_{m}(x)+\mu_{h}^{\lambda} l_{h}^{\lambda} c_{h}(x)\right)}\right]
\end{aligned}
$$

where $Q$ stands for sensor characteristics. $E_{s}$ and $E_{d}$ are intensities of specular and diffuse components of light source, respectively. $w_{s}$ is the specular factor giving rise of highlight, which is the dot product $\left(w_{S}(x)=\vec{V} \overrightarrow{S(x)}\right)$ between viewer direction $(\vec{V})$ and specular reflection direction $(\vec{S})$. $\alpha$ is a material relevant constant, which assigns large value for a smooth surface while small value for a rough one. $w_{d}$ is wavelength-independent shading variation due to scene geometry, which is the dot product $\left(w_{d}(x)=\right.$ $\overrightarrow{N(x)} \vec{L})$ between surface normal $(\vec{N})$ and lighting direction $(\vec{L}) \cdot \mu_{m}^{\lambda}$ and $\mu_{h}^{\lambda}$ are wavelength-dependent absorptive coefficients of melanin and hemoglobin, respectively. $l_{m}^{\lambda}$ and $l_{h}^{\lambda}$ are the lengths of light path in epidermis and dermis layers. $c_{m}$ and $c_{h}$ are densities of melanin and hemoglobin in a sampled volume of skin.

\section{METHODS}

\section{A. Specular Reflection and Diffuse Reflection Separation}

Based on the specular+diffuse reflectance model, Yang et al. [6] estimate the maximum diffuse image and apply it as intensity guidance to remove specular reflection meanwhile keeping edges between surface materials having different hue values. This method works well on toy images with smooth surface [6]. But it poses much difficulty when applied directly on texture-rich skin images, since surface texture (e.g. skin line patterns), in skin areas sharing similar hue but different saturations, will be filtered out as specular component. Subtracting it from spectral image, the diffuse component turns out blurred, and part of texture information, having diagnostic importance for skin disease analysis, is lost. These drawbacks can be clearly detected in the specular component and diffuse image of a malignant melanoma (MM) calculated by [6] in Fig. 1(b) and Fig. 1(d).

Thus in this study, we apply Yang's method [6] to localize the candidate specular reflection areas first. Then the highlight regions are decided by selecting pixel intensity larger than a preset threshold in the candidate specular map, and this value is set to 20 in this work. Compared to the candidate specular component in Fig. 1(b), surface texture information is greatly decreased in the highlight image after thresholding (Fig. 1(c)).

In principle, the diffuse reflection can be obtained by subtracting specular component from spectral skin image. But it should be noted that there are some fake intensity pixels, visible as dark spots, existing in the derived diffuse image (Fig. 1). This is because image intensity of these pixels is dominated by the specular reflection, while diffuse information is almost missing due to local surface smoothness and observation direction. Subtraction results in fake pixel intensity in the RGB channels, but not the real diffuse chromaticity. In order to avoid this problem, the diffuse chromaticity of the detected specular pixels is defined by the mean value of neighboring points using an interpolation approach. Fig. 1(f) shows the diffuse image after specular reflection removal by our method.

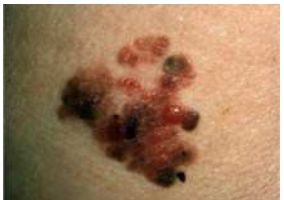

(a)

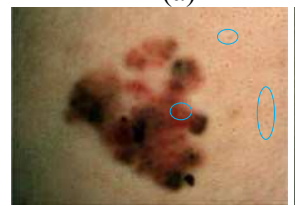

(d)

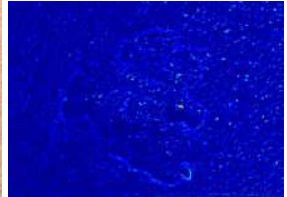

(b)

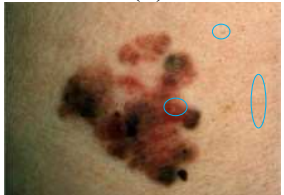

(e)

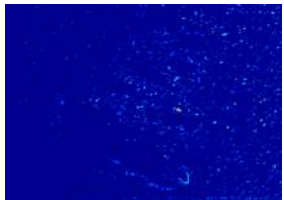

(c)

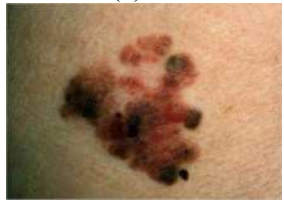

(f)
Fig. 1 Specular reflection removal in skin color image. (a) An RGB melanoma image from a public database [7] having highlight and shading as artifacts. (b) Candidate specular reflectance component by original Yang's method [6]. (c) Specular reflectance component after thresholding. (d) Diffuse image by subtracting specular reflection (b) from (a), pointing out the areas having fake intensity pixels by ellipses. (e) Diffuse image by subtracting specular reflection (c) from (a), pointing out the areas having fake intensity pixels by ellipses. (f) Diffuse image by pixel interpolation of our method.

\section{B. Decomposition of Diffuse Reflection}

After specular reflection removal, only diffuse reflection remains. Hence, image diffuse reflection can be formulated by a linear combination of chromophore coefficients, optical parameters of light source and detector, as well as shading effects in an inverse logarithmic form:

$$
\begin{aligned}
I_{d}(x, \lambda)= & 2\left(\mu_{m}^{\lambda} l_{m}^{\lambda} c_{m}(x)+\mu_{h}^{\lambda} l_{h}^{\lambda} c_{h}(x)\right)-\log Q(\lambda)- \\
& \log E_{d}(\lambda)-\log w_{d}(x)
\end{aligned}
$$

Bilateral filter, initially proposed in [8], was developed for image denoising meanwhile preserving important edges and features. It extends the concept of Gaussian smoothing through a weighted average process of neighboring pixels. Applying bilateral filter on the inverse logarithmic diffuse skin image, the output intensity $I_{\mathrm{d}}^{\prime}(x, \lambda)$ at pixel $x$ is:

$I_{d}^{\prime}(x, \lambda)=\frac{1}{A} \sum_{p \in \Omega_{x}} f(p, x) g\left(I_{d}(p, \lambda), I_{d}(x, \lambda)\right) I_{d}(p, \lambda)$

$=\left[\frac{1}{A} \sum_{p \in \Omega_{x}} f(p, x) g\left(I_{d}(p, \lambda), I_{d}(x, \lambda)\right)\left(2 \mu_{m}^{\lambda} l_{m}^{\lambda} c_{m}(p)+\right.\right.$

$\left.2 \mu_{h}^{\lambda} l_{h}^{\lambda} c_{h}(p)-\log w_{d}(p)\right]+\left[-\log E_{d}(\lambda)-\log Q(\lambda)\right]$

where $p$ belongs to the neighborhood $\left(\Omega_{\mathrm{x}}\right)$ of pixel $\mathrm{x}$, and $A$ is a normalizing constant. The first term contains chromophore reflectance $\left(\mu^{\lambda} l^{\lambda} c(x)\right)$ and shading effects $\left(w_{d}(x)\right)$, whose contributions in $I_{d}^{\prime}$ are controlled by spatial function $f$ and range function $g$, defined by Gaussian kernels as in Equation (4). The second term corresponds to illumination information, which is a function of $\lambda$.

$$
f(p, x) g\left(I_{d}(p), I_{d}(x)\right)=\exp \left(-\frac{\|p-x\|^{2}}{2 \sigma_{1}^{2}}\right) \exp \left(-\frac{\left\|I_{d}(p)-I_{d}(x)\right\|^{2}}{2 \sigma_{2}^{2}}\right)
$$

Considering that shading is usually a low frequency component which changes gradually across large skin areas, high frequency chromophore elements can be smooth out by 
selecting a relative larger intensity standard deviation $\sigma_{2}$, whose value depends on specific applications. Subtracting $I_{d}^{\prime}(x, \lambda)$ from $I_{d}(x, \lambda)$, intrinsic chromophore information can be obtained. We then embed this bilateral filtering in an iterative process such that chromophore reflectance will iteratively propagate until the difference between images before and after filtering is smaller than a preset threshold $t$ for every pixel. In this study, $t=0.01 * \ln (255) \approx 0.05$, which corresponds to $1 \%$ of overall image intensity range.

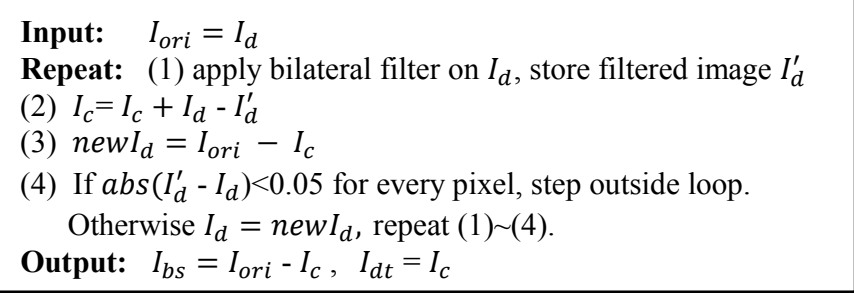

Therefore, a skin image can be decomposed into a base layer having low frequency imaging factors, and a detail layer containing chromophore reflectance with a small part of high frequency wavelength-independent artifacts $\left(\epsilon_{x}\right)$ :

$$
\begin{gathered}
I_{\lambda, b s}=-\log Q(\lambda)-\log U(\lambda)-\log w_{d}(x) \\
I_{\lambda, d t}=\mu_{m}^{\lambda} l_{m}^{\lambda} c_{m}(x)+\mu_{h}^{\lambda} l_{h}^{\lambda} c_{h}(x)+\epsilon_{x}
\end{gathered}
$$

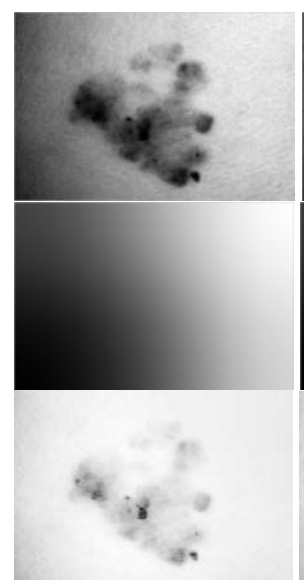

(a)

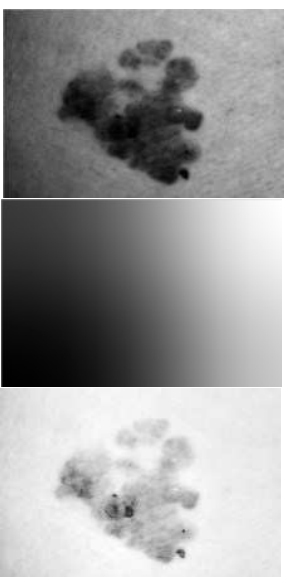

(b)

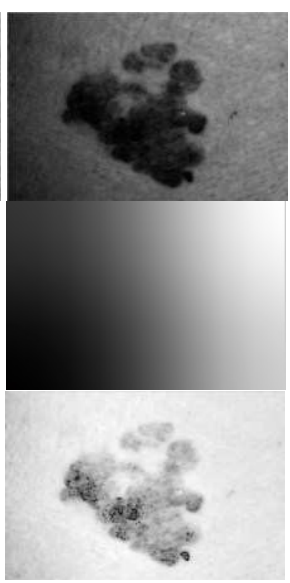

(c)
Fig. 2 Base layers and detail layers separation of the diffuse melanoma image in Fig. 1(f). (a) red (b) green and (c) blue channels. $1^{\text {st }}-3^{\text {rd }}$ rows: the diffuse images, the base layers, and the detail layers by the bilateral decomposition, respectively.

Fig. 2 shows the base layers and detail layers derived from the diffuse skin image in Fig. 1(f). It is obvious that direct division between RGB channels cannot properly address shading effects, because spectral responses at different wavelength bands show varied illumination information. The proposed bilateral decomposition computes low frequency components through a nonlinear weighted average process of neighboring pixels. Hence the detected base layers, responsible for varied lighting intensity across large smooth regions, match with the content of original spectral images. The resultant detail layers show pigmentation in large skin regions with little illumination and shading influence.
It is worth noting that the exact degree of pigmentation in the detail layers greatly changes among the RGB channels. From Fig. 3, the absorbance spectrum of melanin and hemoglobin varies with wavelength. The diffuse reflectance of skin in a specific spectral band can then be considered as an effect attributed to particular chromophores. Therefore, melanin and hemoglobin indices can be calculated.

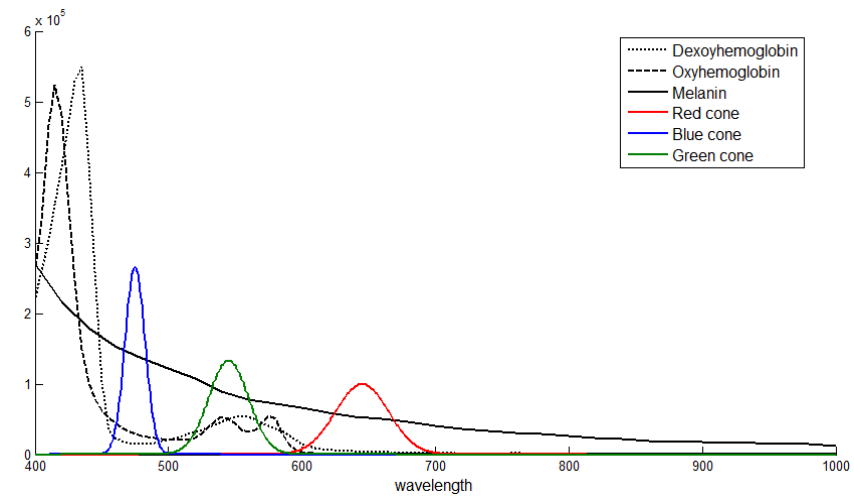

Fig. 3 Spectral absorption of major chromophores from 400nm to $1,000 \mathrm{~nm}$ based on published data [9], and their relation with spectral responses of conventional RGB digital cameras.

\section{Melanin Index and Hemoglobin Index Approximation}

As shown in Fig. 3, melanin, the major pigmentation chromophore, effectively absorbs light from $400 \mathrm{~nm}$ to 1,000nm; wheras oxyhemoglobin and deoxyhemoglobin, the major blood chromophores, both greatly absorb light around $450 \mathrm{~nm}$ and $570 \mathrm{~nm}$, respectively. Due to the increased spectrum attenuation of hemoglobin, the absorption of longer wavelength light $(>620 \mathrm{~nm})$ is dominated by melanin, whilst that of hemoglobin is negligible. Associating chromophore absorbance with the spectral responses of conventional RGB cameras, image intensity in red channel $(\sim 650 \mathrm{~nm})$ is primarily attributed to melanin concentration, while those of green $(\sim 550 \mathrm{~nm})$ and blue $(\sim 450 \mathrm{~nm})$ channels are the joint effects of melanin and hemoglobin, simultaneously. The detail layers of skin image intensity in the RGB channels can then be expressed as:

$$
\begin{gathered}
I_{r, d t}=\mu_{m}^{r} l_{e p}^{r} c_{m}(x)+\epsilon_{x} \\
I_{g, d t}=\mu_{m}^{g} l_{e p}^{g} c_{m}(x)+\mu_{h}^{g} l_{d}^{g} c_{h}(x)+\epsilon_{x} \\
I_{b, d t}=\mu_{m}^{b} l_{m}^{b} c_{m}(x)+\mu_{h}^{b} l_{h}^{b} c_{h}(x)+\epsilon_{x}
\end{gathered}
$$

From Fig. 2, the effects of low frequency imaging factors can be eliminated by removing the base layer from diffuse spectral image. While the influence of high frequency wavelength-independent artifacts $\left(\epsilon_{\mathrm{x}}\right)$ can be compensated by subtracting the detail layers between RGB channels:

$$
\begin{gathered}
I_{g, d t}-I_{r, d t}=\left(\mu_{m}^{g} l_{m}^{g}-\mu_{m}^{r} l_{m}^{r}\right) c_{x, m}+\mu_{h}^{g} l_{h}^{g} c_{x, h} \\
I_{b, d t}-I_{r, d t}=\left(\mu_{m}^{b} l_{m}^{b}-\mu_{m}^{r} l_{m}^{r}\right) c_{x, m}+\mu_{h}^{b} l_{h}^{b} c_{x, h} \\
I_{b, d t}-I_{g, d t}=\left(\mu_{m}^{b} l_{m}^{b}-\mu_{m}^{g} l_{m}^{g}\right) c_{x, m}+\left(\mu_{h}^{b} l_{h}^{b}-\mu_{h}^{g} l_{h}^{g}\right) c_{x, h}
\end{gathered}
$$

Considering that absorptive coefficients and light penetration lengths can be determined based on the previous 
publications $[9,10]$, the melanin density and hemoglobin density can be estimated as:

$$
\left(\begin{array}{l}
c_{m} \\
c_{h}
\end{array}\right)=\left(\begin{array}{ccc}
0.176 & 0.747 & 1.042 \\
0.086 & 0.352 & -0.488
\end{array}\right) *\left(\begin{array}{c}
I_{g, d t}-I_{r, d t} \\
I_{b, d t}-I_{r, d t} \\
I_{b, d t}-I_{g, d t}
\end{array}\right)
$$

In this study, the melanin index is approximated by $M I=c_{m}$ and the hemoglobin index is $H I=c_{h}$.

Fig. 4 shows the MI and $\mathrm{HI}$ of the MM having both dark brown/black and reddish responses that reflect the underlying melanin and hemoglobin densities. The MI mapping successfully addresses the effect of shading and reveals dark brown areas that are concealed by illumination in the ICA method. Its corresponding HI mapping gives apparent contrast between blackish and reddish areas within MM, and regions of surrounding healthy skin. All these should result in more accurate MM diagnosis, compared to the $\mathrm{MI}$ and $\mathrm{HI}$ obtained from the ICA method [2].

\section{EXPERIMENTS AND RESULTS}

\section{A. Melanoma Diagnosis Using Chromophore Descriptors}

In order to quantitatively verify the goodness of the proposed method, chromophore descriptors based on MI and $\mathrm{HI}$ are used as the only diagnostic features for MM diagnosis. Specifically, (1) after a few preprocessing (hair removal and lesion segmentation), $\mathrm{MI}$ and $\mathrm{HI}$ are calculated, and a third chromophore index $C I=0.5(M I+H I)$ is generated to represent the joint pigmentation status. (2) For each greyscale mapping, color range is quantized into 16 bins, and the absolute color variation of isolated lesion is defined as $C V_{l, a b v}=\sqrt{\sum_{i=0}^{15}\left(q_{i}-\bar{q}\right)^{2} \frac{N_{i}}{N}}$. Here $q_{i}$ and $\bar{q}$ are the mean color values of a quantized bin $i$ and the overall lesion respectively, $N_{i}$ and $N$ donate the corresponding pixel numbers. (3) Similarly computing the absolute color variation of normal skin $C V_{s, a b v}$, the relative color variation $C V_{l, r l v}=C V_{l, a b v}-C V_{s, a b v}$ is obtained. (4) A 12D diagnostic feature vector is finally constructed, by associating additional pigmentation asymmetry measures [11].

A number of 197 conventional RGB skin lesion images (62 MMs, $135 \mathrm{BN})[7,12]$ is collected to form an experimental dataset. A linear support vector machine is used as the classifier, and a 10-fold cross validation is the trainingtesting strategy [11].

As shown in Table 1, chromophore descriptors from the bilateral decomposition and an ICA method [2] both improve the automatic MM diagnosis, compared to the results based on RGB colorimetry. Considering that the most important objective for MM diagnosis is to maximize the correct recognition rate of malignant lesions, the best classification is achieved by experiment using chromophore descriptors from the bilateral decomposition, which gives $87.86 \%$ sensitivity, $86.05 \%$ specificity, and $86.62 \%$ overall diagnostic accuracy, respectively.

Table 1. Melanoma diagnosis using color descriptors based on RGB colorimetry, the ICA method [2], and the proposed bilateral decomposition. AUC: areas under the receiver operating curve.

\begin{tabular}{|l|l|l|l|}
\hline & RGB & ICA & Bilateral \\
\hline Sensitivity (\%) & 71.85 & 77.23 & $\mathbf{8 7 . 8 6}$ \\
\hline Specificity (\%) & $\mathbf{8 9 . 2 7}$ & 88.67 & 86.05 \\
\hline Accuracy (\%) & 83.79 & 85.07 & $\mathbf{8 6 . 6 2}$ \\
\hline AUC & 0.8154 & 0.8627 & $\mathbf{0 . 9 2 0 3}$ \\
\hline
\end{tabular}

\section{B. Differentiation of Inflammation and Hyperpigmentation in Acne Skin Images}

Acne vulgaris, a highly prevalent skin disease, is believed resulting from proliferation of propionibacterium acnes in pilosebaceous unit [13]. It can lead to inflammatory acne (visible as red papules) due to increase of oxyhemoglobin level. Dark scars, known as secondary acne hyperpigmentation, will appear thereafter thanks to excess melanin production during the healing process [13].

In this section, acne segmentation is presented as a qualitative evaluation to show the usefulness of the proposed method for another skin disease analysis. In the experiment, (1) the combined mapping $C I$ is used as the only pigmentation descriptor and forwarded to a Markov random field model [14] to isolate acne from surrounding normal skin. (2) The segmentation mask is then projected back to normalized MI and HI, respectively. (3) If the average $\mathrm{HI}$ value of a detected acne area is larger than that of MI, acne is classified as inflammatory, otherwise it is hyperpigmentation.

A number of 50 challenging RGB acne images [12], captured under uncontrolled environment, have been tested in this study. Two examples, having highlight and shading as artifacts, are shown in Fig. 5. Compared to the ICA approach, pigmentation mappings based on the bilateral decomposition are more robust to external imaging factors, and the detected acne areas are more accurate according to human visual assessment. Moreover, the segmented inflammatory acne and hyperpigmentation scar in Fig. 5(c)(e) inversely correspond to pigmentation distributions in $\mathrm{HI}$ and MI, respectively. But this discrimination will not be

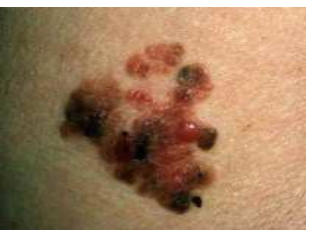

(a)

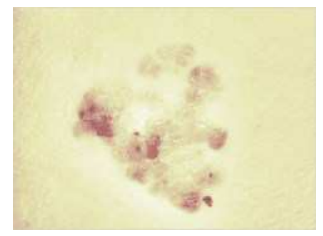

(b)

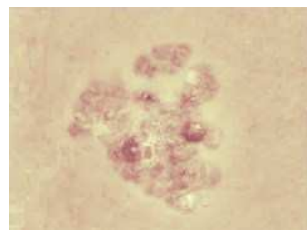

(c)

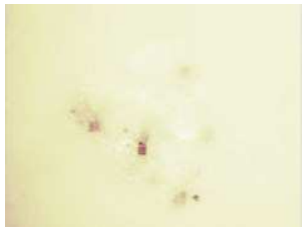

(d)

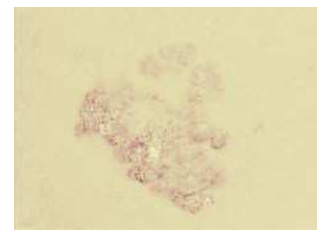

(e)

Fig. $4 \mathrm{MI}$ and $\mathrm{HI}$ of the MM image in Fig. 1, calculated by different chromophore extraction algorithms. (a) original image. (b) MI and (c) HI from the bilateral decomposition. (d) the first and (e) the second independent component calculated by the ICA method [2]. 


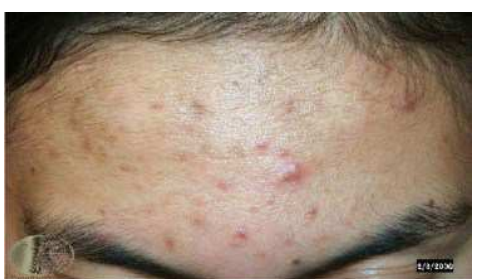

(a)

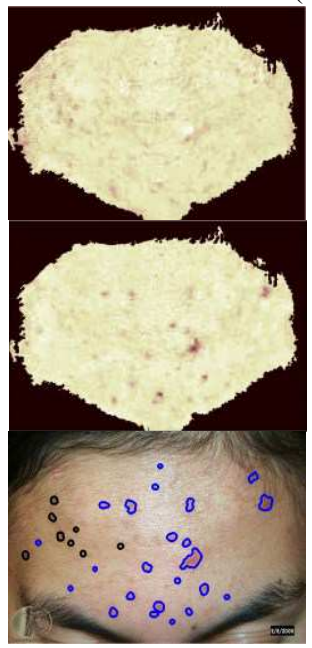

(c)

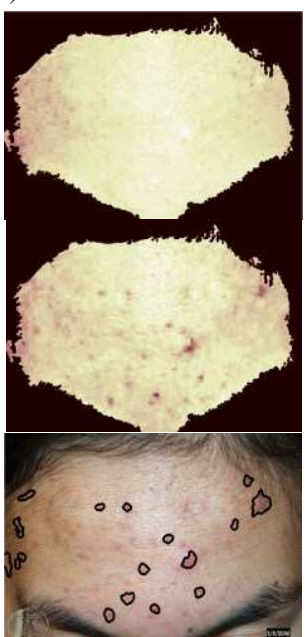

(d)

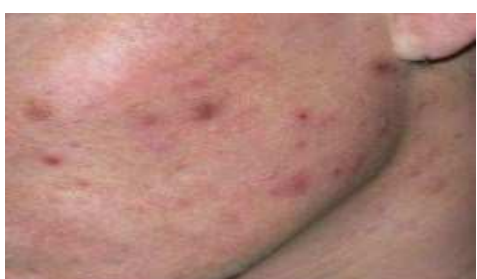

(b)

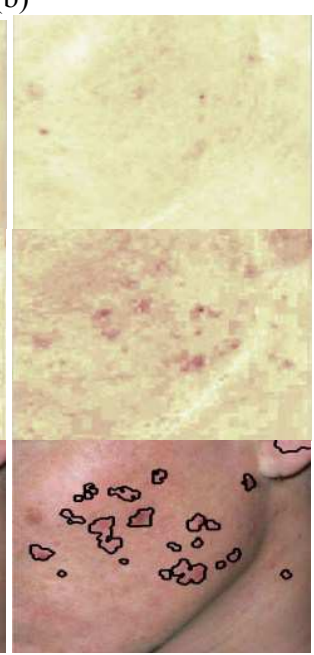

(f)

Fig. 5 Acne segmentation of two conventional RGB images using chromophore descriptors derived from different methods. (a)(b) Original images with highlight/shading. (c)(e) Results by the bilateral decomposition. From top to bottom, MI mappings, HI mappings, and corresponding segmentation results highlighting the inflammatory acne (blue) and hyperpigmentation (black). (d)(f) Results by the ICA method [2]. From top to bottom, two independent components, and corresponding segmentation results.

available for the ICA method, since MI and HI should be identified by human assessment.

\section{CONCLUSION}

This paper proposes a novel chromophore extraction algorithm using bilateral decomposition. The derived melanin and hemoglobin indices are well identified, and prove robust to highlight and shading in skin color images captured under uncontrolled imaging environment. Experiments show that the proposed method increases automatic melanoma diagnostic accuracy, and exhibits a high potential for other skin disease analysis. For the next step study, the accuracy of acne segmentation by the proposed method will be quantitatively evaluated and reported, when professional assessments, by an experienced dermatologist currently under process in a collaborating hospital, are available.

\section{ACKNOWLEDGMENT}

This research study has been conducted within the LIRA Consortium under the funding of INRIA-DPE fellowship.

\section{REFERENCES}

[1] B. Wandell. Foundations of vision, Sinauer Associates, 1995.

[2] N. Tsumura, H. Haneishi, Y. Miyake. "Independent component analysis of spectral absorbance image in human skin," Opt. Rev., vol.7, pp. 479-82, 2000.
[3] A. Madooei, M. Drew, M. Sadeghi. M. Atkins. "Intrinsic Melanin and Hemoglobin Color Components for Skin Lesion Malignancy Detection," In proc. of 15th Medical Image Computing and Computer Intervention (MICCAI), vol. 7510, pp. 315-322, Nice, 2012.

[4] E. Claridge, S. Cotton, P. Hall, M. Moncrieff. "From color to tissue histology: Physics-based interpretation of images of pigmented skin lesions,” Med. Image Anal., vol. 7, pp. 489-502, 2003.

[5] T. Yamamoto, H. Takiwaki, H. Ohshima. "Derivation and clinical application of special imaging by means of digital cameras and image freeware for quantification of erythema and pigmentation," Skin Res. Technol., vol. 14, pp. 26-34, 2008.

[6] Q. Yang, S. Wang, N. Ahuja. "Real-time Specular Highlight Removal Using Bilateral Filtering," In proc. of 11th European Conference on Computer Vision (ECCV), vol. IV, pp. 87-100, Greece, 2010.

[7] Dermatology Online Atlas, http://www.dermis.net/.

[8] C. Tomasi, R. Manduchi. "Bilateral Filtering for gray and color images," In: 6th international Conference on Computer Vision (ICCV), pp. 839-0846, New Delhi, 1998.

[9] S. Jacques. "Skin optics," Oregon Medical Laser Center News, January, 1998.

[10] G. Keller, V. Lacombe. P. Lee, J. Watson. Lasers in Aesthetic Surgery, Thieme, 2001.

[11] Z. Liu, J. Sun, L. Smith, M. Smith, R. Warr. "Distribution quantification on dermoscopy images for computer-assisted diagnosis of cutaneous melanoms," Med. Biol. Eng. Comput., vol. 50, no. 5, pp. 503-513, 2012.

[12] Dermnet NZ, http://www.dermnetnz.org/.

[13] G. Webster. "Inflammation of acne vulgaris," J. Am. Acad. Dermatol., vol. 33, pp. 247-253, 1995.

[14] Z. Kato, J. Zerubia. Markov random fields in image segmentation, Foundations and Trends in Signal Processing, 2012. 